\title{
ASOCIACIÓN ENTRE ADIPONECTINA Y EL SÍNDROME DE OVARIO POLIQUÍSTICO: REVISIÓN DE LA LITERATURA
}

\section{Association between adiponectin and polycystic ovary syndrome: review of the literature}

Carlos Bustamante-Zuluaga, $M D^{1}$; Felipe Vergara-Quintero, $M D^{I}$; Israel Díaz-Yúnez, $M D^{2}$

Recibido: octubre 12/12 - Aceptado: junio 11/13

\section{RESUIMEN}

Objetivo: evaluar la literatura existente respecto a la asociación entre la adiponectina y el síndrome de ovario poliquístico (SOP).

Materiales y métodos: se realizó una búsqueda bibliográfica en las bases de datos electrónicas ScieLO, LILACS, PubMed vía ovid, así como de revistas indexadas y sociedades médicas reconocidas tales como Fertility and Sterility, The European Journal of Endocrinology, The Journal of Biological Chemistry, Human Reproduction y Nature Medicine.

Resultados: se revisaron 75 títulos, de los cuales 50 cumplían con nuestros criterios de selección, correspondientes a diseños de tipo transversal, casos y controles, y un metaanálisis. Los niveles de adiponectina sérica fueron menores en mujeres con síndrome de ovario poliquístico. Los resultados respecto a los niveles de adiponectina y la obesidad son contradictorios en mujeres con y sin SOP. Es escasa la información respecto a esta asociación en la población latinoamericana.

1 Ginecoobstetra, Instituto de Reproducción Humana (PROCREAR). Barranquilla, Colombia.

2 Coordinador, Departamento de Investigación del Instituto de Reproducción Humana (PROCREAR). Barranquilla, Colombia. auditor@hotmail.com
Conclusión: la literatura muestra que hay una asociación entre los niveles bajos de adiponectina y las mujeres que presentan síndrome de ovario poliquístico, independientemente de la presencia o no de obesidad.

Palabras clave: adiponectina, obesidad, hiperandrogenismo, adipocitoquinas, insulino-resistencia, síndrome de ovario poliquístico.

\section{ABSTRACT}

Objective: To review the existing literature on the association between adiponectin and polycystic ovary syndrome (POS).

Materials and methods: A search of the literature was conducted in the Scielo, LILACS and PubMed electronic databases via OVID, as well as in indexed journals and recognized medical societies such as Fertility and Sterility, The European Journal of Endocrinology, The Journal of Biological Chemistry, Human Reproduction and Nature Medicine.

Results: Overall, 75 titles were reviewed and 50 were found to meet our selection criteria. They included cross-sectional, case-controls studies and one meta-analysis. Serum adiponectin levels were lower in women with polycystic ovary syndrome. Results regarding adiponectin levels and obesity are conflicting in women with and without POS. 
There is little information about this association in the Latin American population.

Conclusión: The literature shows an association between low adiponectin levels and women with polycystic ovary syndrome, regardless of the presence or absence of obesity.

Keywords: Adiponectin, obesity, hyperandrogenism, insulin resistance, polycystic ovary syndrome.

\section{INTRODUCCIÓN}

Descripción de la condición: el síndrome de ovario poliquístico (SOP) es el trastorno endocrino más frecuente en las mujeres en edad reproductiva (1). Su prevalencia es de 4-8\% y tan solo en Estados Unidos se considera que afecta entre 7 a 10 millones de mujeres $(1,2)$. En la actualidad no existe una definición que sea aceptada por todos los clínicos. Se han realizado varias reuniones de consenso, con la intención de lograr un acuerdo sobre cómo describir este síndrome (3-5), definir el abordaje de la fertilidad en estas pacientes (6) e identificar las brechas de conocimiento respecto a la salud de las mujeres en el $\operatorname{SOP}(7,8)$. El diagnóstico más utilizado hoy en día sigue siendo con base en los criterios de Rotterdam, los cuales toman en cuenta la presencia de dos de los siguientes parámetros: anovulación crónica, signos clínicos y bioquímicos de hiperandrogenización y ovarios poliquísticos en ecografía (4).

Hasta el día de hoy no se conoce de manera completa por qué se produce el síndrome de ovario poliquístico, aunque se han descrito varias teorías para explicar la fisiopatología de este desorden destacándose entre estas: a) la elevación de los niveles de hormona luteinizante ( $\mathrm{LH}$ ) y b) la elevación de los niveles de insulina (9). En el primer caso, el aumento de pulsaciones de LH a nivel hipofisario se traduce en una mayor oferta de LH que al actuar sobre sus receptores en las células de la teca incrementan la degradación del colesterol y posterior esteroidogénesis. Adicionalmente, la hiperestrogenemia produce un aumento de retroalimentación positiva sobre la LH contribuyendo aún más al aumento de andrógenos.

En cuanto a la hiperinsulinemia asociada al SOP, probablemente es el resultado entre la secreción aumentada de la insulina basal, alteraciones en las señales insulino-receptor y una disminución de la depuración de la insulina hepática (10). Es posible que en las mujeres con SOP la hiperinsulinemia provoque niveles altos de andrógenos ocasionando a nivel ovárico atresia folicular e incapacidad para la formación de estrógenos. En este contexto, la hiperestimulación ovárica por la insulina provocaría hiperandrogenismo gracias a procesos como: 1) el incremento de las enzimas $P 450 \mathrm{c}-17 \alpha$ a nivel de las células de la teca en los folículos ováricos (intermediarias principales en la conversión de progesterona a 17-hidroxiprogesterona y de esta a androstendiona); 2) aumento de la $5 \alpha$ reductasa a nivel de piel, lo cual incrementaría la conversión de testosterona y la disminución de la actividad de la aromatasa; 3) aumento de IGF-1 (factor de crecimiento insulínico tipo I, a nivel sérico e intrafolicular), el cual sinergiza la acción de la LH e indirectamente activa las enzimas del citocromo P450 17 $\alpha$ hidroxilasa $(10,11)$. Además, la hiperinsulinemia inhibe la producción de la proteína transportadora de hormonas sexuales (SHBG), lo cual aumenta la proporción de testosterona libre que a su vez representa la forma activa de la hormona (11). Adicionalmente, la conversión periférica de andrógenos a estrona inhibe la secreción de FSH llevando a ciclos anovulatorios (12).

Descripción de la exposición asociada: el tejido adiposo es considerado en la actualidad como una glándula endocrina verdadera, ya que cumple con una gran variedad de funciones que regulan distintos procesos fisiológicos y metabólicos en el organismo (13).Dentro de este tejido tenemos que las adipocitoquinas cumplen un papel importante en muchos mecanismos de regulación de la fisiología humana. Las adipocitoquinas son péptidos producidos por la célula base del tejido adiposo, es decir, el adipocito (14). Estas se secretan para actuar en forma local (autocrina/paracrina) y de manera sistémica (endocrina) (15). Pero bien sea a corta o larga distancia, las células endocrinas son 
controladas por estímulos externos que generan un mecanismo de retroalimentación (16). Los sitios más importantes donde ejercen sus acciones son: el sistema musculoesquelético, el sistema nervioso, el hígado, el hueso, el sistema cardiaco, entre otros $(16,17)$.

La adiponectina hace parte de este gran arsenal de sustancias y participa en el mantenimiento de la sensibilidad a la insulina, captación de glucosa y oxidación de ácidos grasos. Esta proteína fue identificada en la década de los noventa por cuatro grupos de investigadores $(18,19,20)$. Está codificada en el brazo largo del cromosoma 3 (locus 3q27), se compone por 244 aminoácidos y su peso molecular es de 30kDa (20). Por procesos de hidroxilación y glicosilación se originan múltiples isoformas de adiponectina circulando en plasma como estructura trimérica, hexamérica y polimérica. La forma monomérica carece de actividad biológica. En cambio, las formas estructurales triméricas que circulan en sangre tienen implicaciones funcionales. Por ejemplo, los estímulos metabólicos como la infusión de glucosa o insulina dan como resultado una reducción transitoria y selectiva de los niveles de adiponectina con mayor peso molecular (21).

La acción de la adiponectina está mediada en la periferia por dos tipos de receptores: adipoR1 y adipoR2, los cuales son proteínas integrales de membrana que tienen un extremo amino-terminal interno y un extremo carboxilo-terminal externo (22). La expresión de estos receptores es bastante ubicua, aunque adipoR1 se expresa principalmente en músculo y posee una mayor afinidad por la adiponectina monomérica que por la adiponectina trimérica, mientras que adipoR 2 se expresa prioritariamente en hígado y tiene una afinidad intermedia por ambas formas (23). En condiciones normales, la adiponectina activa las quinasas del AMPc y los receptores PPAR $\alpha$ (peroxisome proliferator-activated receptor $\alpha$ ) tanto en músculo esquelético como en hígado, lo cual conlleva una mayor combustión de ácidos grasos, mayor consumo de energía y disminución de triglicéridos en hígado y en músculo, interviniendo en el proceso normal de la captación de insulina en sus receptores (24).

Como contraparte se ha documentado que el incremento en el contenido tisular de triglicéridos interfiere con la activación de fosfoinositol3-quinasa (PI-3K) por la insulina, lo que a su vez produce una translocación del transportador de glucosa (GLUT-4) a la membrana y captación de la misma glucosa (25). Quiere decir esto que los niveles elevados de triglicéridos producen una disminución en la sensibilidad a la insulina al interferir con los mecanismos de transducción de señales del receptor de esta $(25,26)$.

Por tanto, la función de la adiponectina es regular estos procesos de transducción, aumentando la expresión de moléculas relacionadas con el transporte y la oxidación de ácidos grasos (26). Simultáneamente, existe una disipación de energía a partir de estos ácidos grasos lo que finalmente llevaría a un incremento en el consumo de los mismos y a la disminución de triglicéridos, lo que en teoría beneficiaría la sensibilidad de la insulina en las células y la posterior captación de glucosa, especialmente en el músculo esquelético (24-26).

Importancia de la revisión: varias patologías endocrinas que comparten algunos parámetros comunes como el aumento de la resistencia a la insulina, la obesidad, el hiperandrogenismo y niveles altos de glucosa en sangre guardan estrecha relación con los niveles bajos de adiponectina. Entre estas patologías se encuentran el síndrome metabólico, la diabetes mellitus 2, la obesidad, la enfermedad cardiovascular y, posiblemente, el síndrome de ovario poliquístico (24, 27-30).

En los últimos años se ha intentado relacionar la adiponectina con el síndrome de ovario poliquístico y así poder establecer si los niveles de esta proteína son causa o consecuencia de este trastorno. Además, cabe la pregunta de si la alteración de los niveles de adiponectina solo se presenta en mujeres con SOP y obesidad adjunta, o en mujeres que solo presentan SOP (sin obesidad). Teniendo en cuenta sus acciones de sensibilización insulínica y los niveles bajos en la 
obesidad, es razonable postular que los niveles de adiponectina deberían estar disminuidos en mujeres con síndrome de ovario poliquístico (31). El objetivo de la presente revisión es realizar una búsqueda exhaustiva de la literatura y determinar cuál es el comportamiento que tiene esta proteína en mujeres que padecen el síndrome de ovario poliquístico.

\section{MATERIALES Y MÉTODOS}

A partir de la pregunta sobre la asociación entre los niveles de adiponectina y la presencia del síndrome de ovario poliquístico se realizó una búsqueda bibliográfica en las bases de datos electrónicas Scielo, LILACS, PubMed vía Ovid, con las siguientes palabras clave: adiponectina, obesidad, hiperandrogenismo, adipocitoquinas, insulino-resistencia, síndrome de ovario poliquístico. También se realizó una búsqueda en revistas especializadas en el tema tales como: Fertility and Sterility, The European Journal of Endocrinology, The Journal of Biological Chemistry, Human Reproduction y Nature Medicine. La búsqueda se limitó a los años 1992-2012. Se analizaron los artículos en inglés y en español.

\section{RESULTADOS}

La búsqueda en la base de datos electrónicas arrojó 75 títulos, de los cuales se excluyeron 15 trabajos debido a que no respondían la pregunta planteada de nuestro estudio. Finalmente se seleccionaron 50 artículos divididos de la siguiente manera: 29 revisiones, 17 estudios de casos y controles, 3 estudios de corte transversal y un metaanálisis (figura 1).

Respecto a la asociación entre la adiponectina y el síndrome de ovario poliquístico la literatura revisada muestra consistentemente que los niveles de adiponectina son menores en pacientes con SOP que en pacientes sin SOP $(32,33,34,35,36)$ (tabla 1). Las investigaciones más recientes se centran en aquellas mujeres que presentan el SOP con obesidad adjunta, y los resultados son contradictorios: algunos estudios muestran que las mujeres obesas presentan niveles de adiponectina más bajos cuando se comparan con mujeres con SOP sin obesidad y con mujeres sin SOP $(37,38,39,40,41,42)$ (tabla 2). Sin embargo, es importante mencionar que existen estudios donde se muestra que no hay ninguna diferencia en los niveles de adiponectina total en

\section{Figura 1}

Resultados de la búsqueda de estudios que evalúan la asociación de la adiponectina con el síndrome de ovario poliquístico

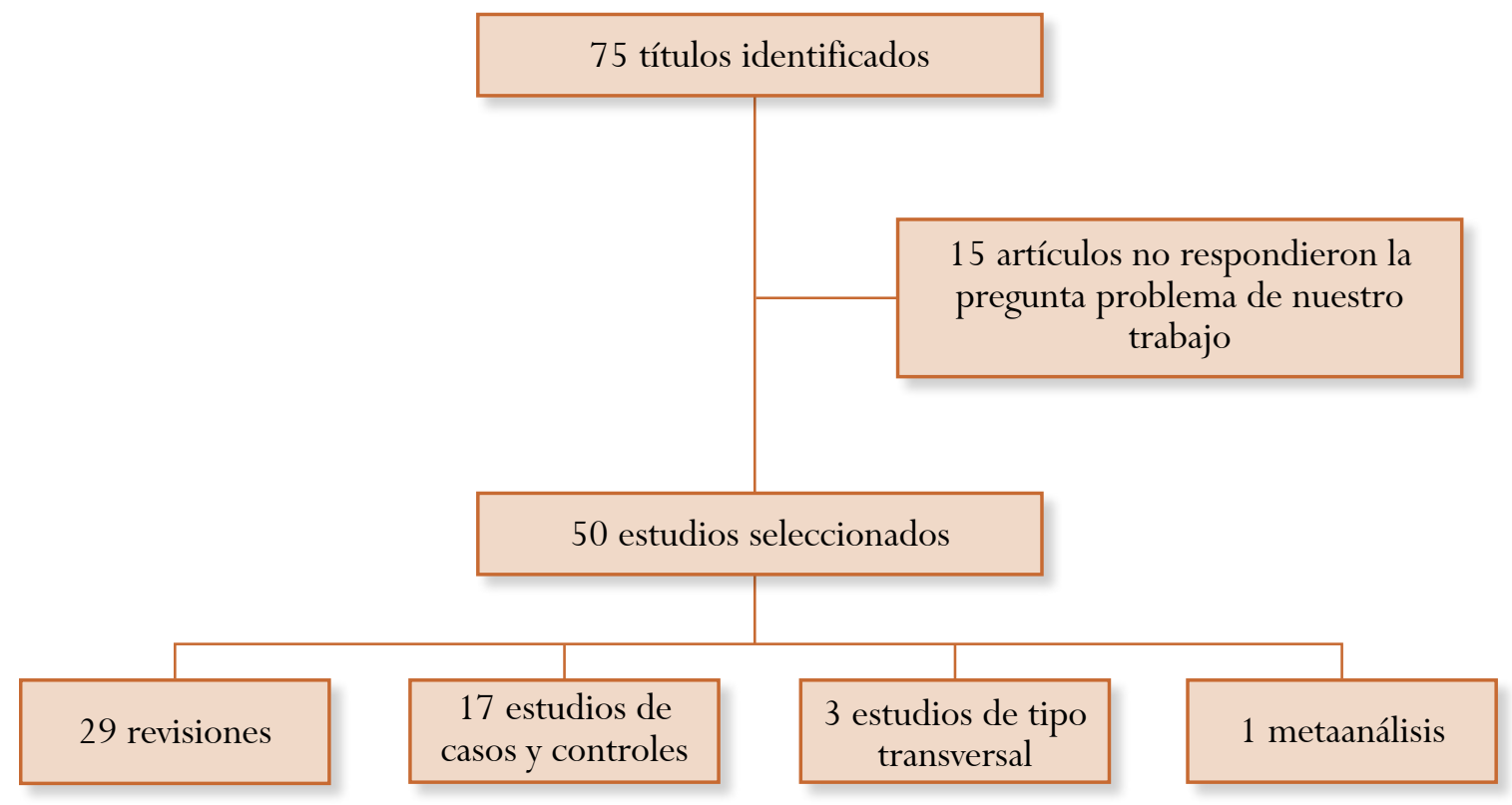




\begin{tabular}{|c|c|c|c|c|}
\hline Autor & Revista & Paciente con SOP & Pacientes control & Valor de $\mathrm{P}$ \\
\hline $\begin{array}{c}\text { Spranger (32) } \\
\text { Alemania }\end{array}$ & $\begin{array}{c}\text { Clinical } \\
\text { Endocrinology } \\
2004\end{array}$ & $7,6 \pm 0,5 \mu \mathrm{g} / \mathrm{ml}$ & $9,8 \pm 0,6 \mu \mathrm{g} / \mathrm{ml}$ & $p=0,006$ \\
\hline $\begin{array}{l}\text { Glintborg (33) } \\
\text { Dinamarca }\end{array}$ & $\begin{array}{c}\text { European Journal of } \\
\text { Endocrinology } \\
2006\end{array}$ & $6,9(2,9-16,4) \mathrm{mg} / \mathrm{l}$ & $7,6(3,0-19,4) \mathrm{mg} / \mathrm{l}$ & $p=0,04$ \\
\hline $\begin{array}{c}\text { Escobar (34) } \\
\text { España }\end{array}$ & $\begin{array}{c}\text { Human Reproduction } \\
2006\end{array}$ & $9,1 \pm 3,5 \mu \mathrm{g} / \mathrm{ml}$ & $11,8 \pm 5,5 \mu \mathrm{g} / \mathrm{ml}$ & $p=0,038$ \\
\hline $\begin{array}{c}\text { Edmon (36) } \\
\text { Canadá }\end{array}$ & $\begin{array}{c}\text { Metabolism } \\
2011\end{array}$ & $3,5(2,47-4,45) \mu \mathrm{g} / \mathrm{ml}$ & $6,0(5,0-6,99) \mu \mathrm{g} / \mathrm{ml}$ & $p=0,001$ \\
\hline $\begin{array}{l}\text { Mazloomi (35) } \\
\text { Irán }\end{array}$ & $\begin{array}{c}\text { International Scholary } \\
\text { Research Network } \\
2012\end{array}$ & $8,4 \pm 2,7 \mathrm{ng} / \mathrm{ml}$ & $13,6 \pm 5 \mathrm{ng} / \mathrm{ml}$ & $p<0,001$ \\
\hline
\end{tabular}

\begin{tabular}{|c|c|c|c|c|c|c|c|c|}
\hline \multirow[t]{2}{*}{ Autor } & \multirow[t]{2}{*}{ Año } & \multicolumn{3}{|c|}{ Población de pacientes (N) } & \multicolumn{3}{|c|}{ Niveles de adiponectina $(\mu \mathrm{g} / \mathrm{ml})$} & \multirow{2}{*}{ Valor de $\mathrm{P}$} \\
\hline & & $\begin{array}{c}\text { SOP y } \\
\text { obesidad }\end{array}$ & $\begin{array}{c}\text { Sin } \\
\text { obesidad }\end{array}$ & Controles & $\begin{array}{c}\text { SOP y } \\
\text { obesidad }\end{array}$ & $\begin{array}{l}\text { SOP sin } \\
\text { obesidad }\end{array}$ & Controles & \\
\hline $\begin{array}{c}\text { Ducluzeau } \\
\qquad(37) \\
\text { Francia }\end{array}$ & 2003 & 8 & 8 & 10 & $6,5 \pm 3,1$ & $8,6 \pm 2,9$ & $13,6 \pm 6,3$ & $p=0,19$ \\
\hline $\begin{array}{l}\text { Orio } \\
(38) \\
\text { Italia }\end{array}$ & 2003 & 30 & 30 & 30 & $21,5 \pm 2,5$ & $35,1 \pm 2,6$ & $29,7 \pm 2,8$ & $p<0,05$ \\
\hline $\begin{array}{l}\text { Carmina } \\
(39) \\
\text { Italia }\end{array}$ & 2005 & 18 & 15 & 14 & $6,9 \pm 0,7$ & $9,2 \pm 1,1$ & $13 \pm 1,5$ & $p<0,05$ \\
\hline $\begin{array}{c}\text { Pinhas-Hamiel } \\
\qquad(40) \\
\text { Israel }\end{array}$ & 2009 & 14 & 16 & 14 & $10,5 \pm 5,5$ & $16,9 \pm 8,0$ & $18 \pm 7,4$ & $p<0,05$ \\
\hline $\begin{array}{l}\text { Dikmen } \\
(41) \\
\text { Turquía }\end{array}$ & 2011 & 25 & 17 & 17 & $23,5 \pm 11,7$ & $30,9 \pm 18$ & $37,8 \pm 20,2$ & $p<0,05$ \\
\hline $\begin{array}{c}\text { Olszanecka } \\
\qquad(42) \\
\text { Polonia }\end{array}$ & 2011 & 19 & 22 & 16 & $10,8 \pm 8,3$ & $21,0 \pm 15,1$ & $26,7 \pm 12,5$ & $p<0,05$ \\
\hline
\end{tabular}


aquellas mujeres con SOP quienes tienen obesidad y mujeres con peso normal, arrojando la duda de si los niveles bajos de adiponectina se disminuyen en presencia de obesidad o únicamente en SOP sin obesidad y otros factores $(29,43)$. En este sentido aporta información el estudio de Yasar et al., en el que se evaluaron jóvenes de una escuela secundaria quienes tenían SOP dividiéndolas en dos grupos: con índice de masa corporal (IMC) menor de 25 $\mathrm{kg} / \mathrm{m}^{2}$ (grupo $1, \mathrm{n}=20$ ) e IMC mayor de $25 \mathrm{~kg} /$ $\mathrm{m}^{2}$ (grupo $2, \mathrm{n}=22$ ). Posteriormente compararon los niveles de adiponectina con un grupo control (sin SOP) también dividiéndolas por IMC menor y mayor de $25 \mathrm{~kg} / \mathrm{m}^{2}$ (grupo 3, $\mathrm{n}=21$ y grupo $4, \mathrm{n}$ $=23$, respectivamente). Los resultados indicaron que los niveles de los grupos 1 y 2 fueron significativamente menores $(8,40 \pm 7,1$ y $6,41 \pm 5,4)$ respecto a los grupos 3 y $4(21,6 \pm 10,5$ y 18,7 $\pm 6,9)$ (44). $\mathrm{Al}$ parecer, según este ensayo, los niveles bajos de adiponectina están más ligados al hecho de tener SOP y no tanto al IMC de las mujeres estudiadas.

Por último, se encontró un estudio realizado en población latinoamericana (45), con base en los cuatro fenotipos más importantes $(\mathrm{A}, \mathrm{B}, \mathrm{C}$ y D) del SOP, de acuerdo con los trabajos de Carmina (46) y basados en los criterios del National Institute of Health (NIH), de la AES y de Rotterdam (47, 48, 49). El objetivo del trabajo fue evaluar las concentraciones de adiponectina en cada fenotipo y correlacionarlas con parámetros metabólicos. Así fue como se demostró que las pacientes que presentaban el SOP clásico, es decir, los fenotipos A y B, tuvieron niveles menores (11 \pm 7 y $12 \pm 8)$ cuando se les comparó con los fenotipos C y D (21 \pm 9 y $17 \pm 4)$, así como con el grupo control $(20 \pm)(p<0,05)$.

\section{DISCUSIÓN}

Esta revisión muestra que hay evidencia que soporta la asociación entre SOP y niveles bajos de adiponectina. En cuanto a su asociación con la obesidad y el SOP la evidencia es contradictoria.
Aunque se ha mencionado que los niveles bajos de adiponectina y los altos índices de resistencia a la insulina parecen ir de la mano, no se pueden considerar característicos absolutos en el SOP. Por otra parte, aún no es claro si los cambios de la adiponectina son consecuencia o causa del SOP

Vale la pena resaltar que la mayoría de las investigaciones involucran tamaños muestrales reducidos, además una importante cantidad de los estudios evaluados incluyeron mujeres con franca obesidad (con y sin SOP) mas no así con otros signos de hiperandrogenización como acné, hirsutismo y niveles altos de testosterona, por tanto no toman en cuenta aquellas pacientes que presentan otros fenotipos de SOP que no son tan comunes. La información en nuestro medio latinoamericano es escasa, la mayoría de investigaciones vienen de distintos países de Europa, por lo tanto limita la generalización de estos resultados a nuestra población.

Implicaciones para la investigación y la práctica: esta asociación representa una oportunidad para realizar investigación en esta área en nuestro medio. Consideramos que el papel de los niveles de adiponectina en el arsenal diagnóstico en el síndrome de ovario poliquístico deberá ser evaluado en los próximos años, ya que los resultados previos son alentadores. Sin embargo, dada la evidencia revisada, no es claro que sea pertinente en la actualidad solicitar una adiponectina sérica como un laboratorio de rutina en el SOP (50).

\section{CONCLUSIONES}

La evidencia actual demuestra que los niveles bajos de adiponectina están asociados a mujeres que padecen síndrome de ovario poliquístico con y sin obesidad. Esto plantea que esta proteína puede explicar muchos de los procesos que ocurren en este síndrome llegando a ser en los próximos años un parámetro por considerar dentro de los criterios diagnósticos. 


\section{REFERENCIAS}

1. Chang RJ. A practical approach to the diagnosis of polycystic ovary syndrome. Am J Obstet Gynecol. 2004;191:713-17.

2. Diamanti-Kandarakis E, Baillargeon JP, Iuorno MJ, Jakubowickz DJ, Nestler JE. A modern medical quandary: Polycystic ovary syndrome, insulin resistance, and oral contraceptive pills. J Clin Endocrinol Metab. 2003;88:1927-32.

3. Rotterdam ESHRE/ASRM-Sponsored PCOS Consensus Workshop Group. Revised 2003 consensus on diagnostic criteria and long-term health risks related to polycystic ovary syndrome (PCOS). Hum Reprod. 2004;19:41-7.

4. Rotterdam ESHRE/ASRM-Sponsored PCOS Consensus Workshop Group. Revised 2003 consensus on diagnostic criteria and long-term health risks related to polycystic ovary syndrome. Fertil Steril. 2004;81:19-25.

5. Azziz R. Diagnosis of polycystic ovary syndrome: The Rotterdam criteria are premature. J Clin Endocrinol Metab. 2006;91:781-85.

6. Olutunmbi Y, Paley K, English JC III. Adolescent female acne: etiology and management. J Pediatr Adolesc Gynecol. 2008;21:171-76.

7. Fauser BC, Tarlatzis BC, Rebar RW, Legro RS, Balen $\mathrm{AH}$, Lobo R, et al. Consensus on women's health aspects of polycystic ovary syndrome (PCOS): the Amsterdam ESHRE/ASRM-Sponsored 3rd PCOS Consensus WorkshopGroup. Fertil Steril. 2012;97: 28-38.e25.

8. National Institute of Health (NIH). Evidencebased Methodology Workshop on Polycystic Ovary Syndrome. December 3-5, 2012.

9. Poretsky L, Piper B. Insulin resistance, hypersecretion of LH, and a dual-defect hypothesis for the pathogenesis of polycystic ovary syndrome. Obstet Gynecol. 1994;84:613-21.

10. Speroff L, Fritz MA. Anovulation and the Polycystic Ovary, Insulin Resistance, Hyperinsulinemia, and Hyperandrogenism. En: Speroff L, Fritz M. Clinical Gynecologic Endocrinology and Infertility. 7 Edition; 2005. p. 476-83.
11. Poretsky L. Insulin resistance and hyperandrogenism: Update. Endoc Rev 1994;2:26-35.

12. Taylor AE. Polycystic ovary syndrome. Endoc Metab Clin North Am. 1998;27:877-902.

13. Flier JS. Obesity wars: molecular progress confronts an expanding epidemic. Cell Press. 2004;116:337-50.

14. Hotamisligil GS. The role of TNF $\alpha$ and TNF receptors in obesity and insulin resistance. J Intern Med. 1999; 245: 612-25.

15. Ailhaud G, Grimaldi P, Négrel R. Adipose cell diferentiation: a long way to Tipperary. Centre Biochimie, Par Valrose, 06108 Nicecédex 2, France.

16. Wong GW, Wang J, Hug C, Tsao S, Lodish HF. A family of Acrp30/adiponectin structural and functional paralogs. Proc Natl Acad Sci. 2004;101:10302-7.

17. Simha V, Szczepaniak LS, Wagner AJ, DePaoli AM, Garg A. Effect of leptin replacement on intrahepatic and intramyocellular lipid content in patients with generalized lipodystrophy. Diabetes Care. 2003;26:30-5.

18. Hu E, Liang P, Spiegelman BM. AdipoQ is a novel adipose- specific gene dysregulated in obesity. J Biol Chem. 1996;271:10697-703.

19. Nakano Y, Tobe T, Choi-Miura NH, Mazda T, Tomita M. Isolation and characterization of GBP28, a novel gelatin-binding protein purified from human plasma. J Biochem. 1996;120:803-12.

20. Maeda K, Okubo K, Shimomura I, Funahashi T, Matsuzawa Y, Matsubara K. cDNA cloning and expression of a novel adipose specific collagen-like factor, apM1 (AdiPose Most abundant Gene transcript 1). Biochem Biophys Res Commun. 1996;22 1:286-9.

21. Tsao TS, Tomas E, Murrey HE, Hug C, Lee DH, Ruderman NB, Heuser JE, Lodish HF. Role of disulfide bonds in Acrp30/adiponectin structure and signaling specificity. Different oligomers activate different signal transduction pathways. J Biol Chem. 2003;278:50810-17.

22. Kadowaki T, Yamauchi T. Adiponectin and adiponectin receptors. Endocrine Reviews. 2005;26:439-51.

23. Elissondo N, Gómez Rosso L, Maidana P, Brites F. Adiponectina: una adipocitoquina con múltiples funciones protectoras. Acta Bioquímica clínica latinoamericana. 2008:42:17-33. 
24. Hotta K, Funahashi T, Arita Y, Takahashi M, Matsuda M, Okamoto Y, et al. Plasma concentrations of a novel, adipose-specific protein, adiponectin, in type 2 diabetic patients. Arterioscler Thromb Vasc Biol. 2000;20:1595-9.

25. Yamauchi T, Kamon J, Waki H, Terauchi Y, Kubota N, Hara K. The fat-derived hormone adiponectin reverses insulin resistance associated with both lipoatrophy and obesity. Nat Med. 2001;7:941-6.

26. Dominguez C. Adiponectina: el tejido adiposo más allá de la reserva inerte de energía. Revista mexicana de endocrinología y nutrición. 2007;15:149-55.

27. Trujillo ME, Scherer PE. Adiponectin: journey from an adipocyte secretory protein to biomarker of the metabolic syndrome. J Intern Med. 2005;257:167-75.

28. Kumada M, Kihara S, Sumitsuji S, Kawamoto T, Matsumoto S, Ouchi N, et al. Association of hypoadiponectinemia with coronary artery disease in men. Arterioscler Thromb Vasc Biol. 2003;23:85-9.

29. Arikan S, Bahceci M, Tuzcu A, Kale E, Gokalp D. Serum resistin and adiponectin levels in young non-obese women with polycystic ovary syndrome. Gynecological Endocrinology. 2010;26:161-66.

30. Arita Y. Paradoxical decrease of an adipose-specific protein, adiponectin, in obesity. Biochem Biophys Res Commun. 1999;257:79-83.

31. Michalakis KG, Segars JH. The role of adiponectin in reproduction: from polycystic ovary syndrome to assisted reproduction. Fertil Steril. 2010;94:1949-57.

32. Spranger J, Möhlig M, Wegewitz U, Ristow M, Pfeiffer A, Schill T, et al. Adiponectin is independently associated with insulin sensitivy in women with polycystic ovary syndrome. Clinical Endocrinology. 2004;61:738-46.

33. Glintborg D, Andersen M, Hagen C, Frystyk J, Hulstrøm V, Flyvbjerg A, et al. Evaluation of metabolic risk markers in polycystic ovarysyndrome (PCOS). Adiponectin, ghrelin, leptin and body composition inhirsute PCOS patients and controls. Eur J Endocrinol. 2006;155:337-45.

34. Escobar-Morreale HF, Villuendas G, Botella-Carretero JI, Alvarez-Blasco F, Sanchón R, Luque-Ramírez M, et al. Adiponectin andresistin in PCOS: a clinical, biochemical and molecular genetic study. Hum Reprod. 2006;21:2257-65.

35. Mazloomi S, Shafiri F, Hajihosseini R, Kalantari S, Mazloomzadeh S. Association between Hypoadiponectinemia and low serum concentrations of calcium and vitamin $\mathrm{D}$ in women with polycystic ovary syndrome. ISRN Endocrinol. 2012: 1-6.

36. Wickham EP 3rd, Cheang KI, Clore JN, Baillargeon JP, Nestler JE. Total and high-molecular weight adiponectin in women with the polycysticovary syndrome. Metabolism. 2011;60:366-72.

37. Ducluzeau P, Cousin P, Malvoisin E, Bornet H, Vidal H, Laville M, et al. Glucose-to-Insulin Ratio Rather than Sex Hormone- Binding Globulin and Adiponectin Levels Is the Best Predictor of Insulin Resistance in Nonobese Women with Polycystic Ovary Syndrome. The Journal of Clinical Endocrinology \& Metabolism. 2003;88:3626-31.

38. Giallauria F, Orio F, Palomba S, Lombardi G, Colao A, Vigorito C. Adiponectin Levels in Women with Polycystic Ovary Syndrome. The Journal of Clinical Endocrinology \& Metabolism. 2003;88:2619-23

39. Carmina E, Orio F, Palomba S, Cascella T, Longo R, Colao A, et al. Evidence for altered adipocyte function in polycystic ovary syndrome. European Journal of Endocrinology. 2005;152:389-94

40. Pinhas-Hamiel O, Sigal S, Pilpel N, Koren I, Boyko V. Adiponectin levels in adolescent girl with polycystic ovary syndrome (PCOS). Clinical endocrinology. 2009;71:823-27.

41. Dikmen E, Tarkun I, Ozturk F, Arslan B, Canturk Z. Plasma adiponectin and resistin levels in women withpolycystic ovary syndrome: relation to body mass index and insulin resistance. Turk J Med Sci. 2011;41:45-52.

42. Olszanecka-Glinianowicz M, Kuglin D, DabkowskaHuc A, Skalba P. Serum adiponectin and resistin in relation to insulin resistance and markers of hyperandrogenism in lean and obese women with polycystic ovary syndrome. European Journal of Obstetrics \& Gynecology and Reproductive Biology. 2011;154:51-6. 
43. O'Connor A, Phelan N, Kyaw T, Boran G, Gibney J, Roche HM. High-Molecular-Weight Adiponectin is Selectively Reduced in Women with Polycystic Ovary Syndrome Independent of Body Mass Index and Severity of Insulin Resistance. J Clin Endocrinol Metab. 2010;95:1378-85.

44. Yasar L, Ekin M, Gedikbasi A, Erturk A.D, Savan K, Ozdemir A, et al. Serum adiponectin levels in high school girls with polycystic ovary syndrome and hyperandrogenism. Journal of Pediatric and Adolescent Gynecology. 2011;24:90-3.

45. Fux Otta C, Szafryk de Merecían P, Vocos M, Mengual R, Fiol de Cuneo M. Niveles séricos de adiponectina y parámetros metabólicos en diferentes fenotipos del síndrome de ovario poliquístico. VIII Congreso FASEN. Revista Argentina de Endocrinología y Metabolismo. 2010;47:98.

46. Azziz R, Carmina E, Dewailly D, Diamanti-Kandarakis E, Escobar H, Futterweit W, et al. Position statement: Criteria for Defining Polycystic Ovary Syndrome as a Predominantly Hyperandrogenic Syndrome: An Androgen Excess Society Guideline. The Journal of Clinical Endocrinology \& Metabolism. 2006;91:4237-45.

47. Guastella E, Longo R, Carmina E. Clinical and endocrine characteristics of the main polycystic ovary syndrome phenotypes. Fertil Steril 2010;6:21972201.

48. Zawadski J, Dunaif A. Diagnostic criteria for polycystic ovary syndrome. En: Dunaif A, Givens JR, Haseltine FP, Merriam GR, eds. Polycystic ovary syndrome. Boston: Blackwell Scientific; 1992. p. 377-84.

49. Merino P, Schulin-Zuthen C, Codner E. Diagnóstico del síndrome de ovario poliquístico; nuevos fenotipos, nuevas incógnitas. Rev Med Chile. 2009;137:1071-80.

50. Toulis KA, Goulis DG, Farmakiotis D, Georgopoulos NA, Katsikis I, Tarlatzis BC. Adiponectin levels in women with polycystic ovary syndrome: a systematic review and a meta-analysis. Hum Reprod Update. 2009;15:297-307. 